\title{
CHARACTERIZATIONS OF NOETHERIAN AND HEREDITARY RINGS
}

\author{
ZHENG - XU HE
}

\begin{abstract}
We characterize the left Noetherian rings by the existence of decompositions of left modules into direct sums of an injective submodule and a submodule containing no injective submodule except 0 . We also prove that a left Noetherian ring is left hereditary iff the suspension of each left ideal (see [7]) is injective or. equivalently, the above decomposition is unique.
\end{abstract}

0. Let $A$ be an Abelian group. Then $A=D \oplus B$, where $D$ is divisible and $B$ is reduced in the sense of Kaplansky [8]; that is, $B$ has no divisible submodule except 0 . If this holds for left modules over a unitary ring $R$, with "injective" and " $i$-reduced" replacing "divisible" and "reduced", respectively, then $R$ must be left Noetherian. The proof requires the theorem of Eilenberg-Bass (see, e.g., Theorem 20.1 , p. 111, of [2]) which characterizes left Noetherian by the requirement that direct sums of injective left modules are injective. Furthermore, if the decomposition, which will be referred to as an i-decomposition, is unique in the sense that $D$ is the unique injective submodule of $A$ for which $A=D \oplus B$, with $B i$-reduced, then every sum of injective submodules is injective, and hence $R$ must be left hereditary by a theorem of Matlis [9]. At the same time, it will be shown that a left Noetherian ring is left hereditary iff the suspension of each left ideal is injective.

1. Throughout this paper, $R$ denotes a unitary ring.

THEOREM 1. The following conditions are equivalent:

(i) $R$ is left Noetherian.

(ii) The direct sum of any countably infinite family of injective left $R$-modules is injective.

(iii) Any left $R$-module admits an i-decomposition.

(iv) Every injective left $R$-module is a direct sum of indecomposable left $R$-modules.

Proof. The equivalence (i) $\Leftrightarrow$ (ii) is known, and due to Eilenberg-Bass. The implication (i) $\Rightarrow$ (iii) is immediate; and (i) $\Leftrightarrow$ (iv) is the theorem of Matlis [9] and Papp [10]. Let us prove (iii) $\Rightarrow$ (ii). Let $\left\{S_{n} ; n=1,2,3, \ldots\right\}$ be a countably infinite

Received by the editors December 19, 1983 and, in revised form, May 23, 1984.

1980 Mathematics Subject Classification. Primary 13E05; Secondary 18 G05.

$K e v$ words and phrases. Injective module, $i$-reduced module, $i$-decomposition, unique $i$-decomposition, Noetherian ring, left hereditary ring, FP-injective module, suspension of a module. 
family of injective left $R$-modules. Let $D$ and $B$ realize an $i$-decomposition of $S=\oplus_{n=1}^{\infty} S_{n}$. For each positive integer $k, T_{k}=\bigoplus_{n=1}^{k} S_{n}$ is an injective submodule of $S$. $B$ being $i$-reduced, we must have $E\left(T_{k} \cap D\right)=T_{k}$ by which we deduce that $T_{k} \subseteq D$, where $E\left(T_{k} \cap D\right)$ denotes the injective hull (included in $T_{k}$ ) of $T_{k} \cap D$. In this way, $S=D$, which is injective. Q.E.D.

2. For convenience, we will "borrow" some notation from the homotopy theory of modules introduced by B. Eckmann and P. Hilton (for more details, see [7, Chapter $13 ; 6$ and 5]). For any left $R$-module $A$, we define the "suspension" of $A$ to be $S(A)=E(A) / A$, where $E(A)$ denotes the injective hull of $A$. Let $f: A \rightarrow B$ be a morphism of left $R$-modules; we denote by $f \simeq{ }_{i} 0$ the fact that $f$ can be extended to any module containing $A$. We write $\bar{\pi}(A, B)=0$ if, for any $f: A \rightarrow B, f={ }_{i} 0$. We say that a left $R$-module $B$ is $F G$-injective if $\bar{\pi}(A, B)=0$ for any finitely generated left $R$-module $A$. The following properties hold:

(1) $f \simeq{ }_{i} 0$ iff $f$ can be extended to the injective hull $E(A)$ of $A$ or, equivalently, to any injective extension $E$ of $A$.

(2) If $f=g+h$, then $g \simeq{ }_{i} 0$ and $h \simeq_{i} 0$ imply $f \simeq_{i} 0$.

(3) If $f=\alpha \circ \beta$, then either $\alpha \simeq{ }_{i} 0$ or $\beta \simeq{ }_{i} 0$ implies $f \simeq{ }_{i} 0$.

(4) For any injective extension $E$ of $A, S(A)$ is injective (or FG-injective, or FP-injective) iff $E / A$ is.

(5) If $\bar{\pi}(I, B)=0$ for each left ideal $I$ of $R$, then $B$ is injective.

(6) $B$ is FP-injective iff $\bar{\pi}(A, B)=0$ for any finitely presented left $R$-module $A$.

Lemma. Assume that $R$ satisfies:

(*) For any left ideal I of $R, S(I)$ is $F G$-injective.

Then the sum of any FG-injective submodules of a left $R$-module is FG-injective and hence FP-injective.

Proof. Let $\left\{V_{i} ; i \in J\right\}$ be a family of FG-injective submodules of a left $R$-module; we need to show that $\sum_{i \in J} V_{i}$ is FG-injective. We may reduce the problem to the case when $J$ is finite, and so it suffices to consider $J=\{1,2\}$. For any positive integer $m$, let $\mathfrak{F}^{m}$ be the family of all $R$-modules which can be spanned by $m$ elements. We will prove the following by induction on $m$ :

$$
\bar{\pi}\left(F, V_{1}+V_{2}\right)=0 \text { for any } F \in \mathfrak{\Im}^{m} .
$$

Firstly, for $m=1$, let $F=R a \in \mathfrak{F}^{1}$ and let $f: F \rightarrow V_{1}+V_{2} \cdot f(a)=v_{1}+v_{2}$ for some $v_{i} \in V_{i}, i=1,2$. Since $V_{i}$ is FG-injective, there exists $\alpha_{i}: E(R) \rightarrow V_{i}$ such that $\alpha_{i}(r)=r v_{i}$ for all $r \in R$. Let $I=\left\{r \in R ; r\left(v_{1}+v_{2}\right)=0\right\}$; and set $S=E(R) / I$ which is FG-injective by (*). Define $\beta: F \rightarrow S$ and $\alpha: S \rightarrow V_{1}+V_{2}$ by $\beta(r a)=[r]$ $\in S=E(R) / I$ and $\alpha([\bar{r}])=\alpha_{1}(\bar{r})+\alpha_{2}(\bar{r})$ for $r \in R$ and $\bar{r} \in E(R)$. We get $f=$ $\alpha \circ \beta$. Now $\beta \simeq{ }_{i} 0$, because $S$ is FG-injective. So $f \simeq{ }_{i} 0$, and thus $(* *)$ holds for $m=1$.

Next, we prove $(* *)$ for $m>1$, assuming that $(* *)$ is true if $m$ is replaced by $m-1$. Let $F=R a_{1}+R a_{2}+\cdots+R a_{m} \in \mathfrak{F}^{m}$. We need show that $f \simeq_{i} 0$ for $f$ : $F \rightarrow V_{1}+V_{2}$. Consider $f_{1}=f / R a_{1}$. By the inductive hypothesis, $f_{1} \simeq_{i} 0$, i.e. there is $f_{1}^{\prime}: E(F) \rightarrow V_{1}+V_{2}$ such that $f_{1}^{\prime} / R a_{1}=f_{1}$. Let $g=f_{1}^{\prime} / F$ and let $h=f-g$. Then 
$h\left(a_{1}\right)=0$, and so there exists $\alpha: H=F /\left(R a_{1}\right) \rightarrow V_{1}+V_{2}$ such that $h=\alpha \circ p$, where $p: F \rightarrow H=F /\left(R a_{1}\right)$ is the canonical projection. Clearly, $H \in \mathfrak{F}^{m}$; again by the inductive hypothesis, $\alpha \simeq_{i} 0$, whence $h \simeq_{i} 0$. But obviously, $g \simeq_{i} 0$; thus $f=g+$ $h \simeq{ }_{i} 0$. Q.E.D.

As a consequence, if $R$ is a left Noetherian ring verifying (*), then $\sum_{i \in J} V_{i}$ is injective when $V_{i}(i \in J)$ all are.

We also observe that if $\left\{V_{i} ; i \in J\right\}$ is a family of injective submodules of a left module over a left Noetherian ring, and if $\sum_{i \in J} V_{i}$ admits a unique $i$-decomposition, then $\sum_{i \in J} V_{i}$ is injective. At this stage, using a theorem of Matlis [9] (see, e.g., Proposition 10 of [3]), we may conclude

THEOREM 2. The following conditions are equivalent:

(i) $R$ is left Noetherian and left hereditary.

(ii) $R$ is left Noetherian and satisfies (*).

(iii) Any left $R$-module admits a unique i-decomposition.

(iv) Every sum of injective submodules of a left $R$-module is injective.

The equivalence (i) $\Leftrightarrow$ (ii) includes Corollary 14.B of [4].

ACKNOWLEDGEMENT. The author wishes to thank the referee for useful suggestions.

\section{REFERENCES}

1. C. Faith, Algebra I: Rings, modules, and categories, Springer-Verlag, Berlin, Heidelberg, and New York, 1973; corrected reprint, 1981.

2. __ Algebra I: Ring theory, Springer-Verlag, Berlin and New York, 1976.

3. Lectures on injective modules and quotient rings, Lecture Notes in Math., Vol. 49, Springer-Verlag, Berlin and New York, 1967.

4. On hereditary rings and Boyle's conjecture, Arch. Math. 27 (1976), 113-119.

5. Zheng-Xu He, Some results on homotopy theory of modules, Rend. Accad. Naz. Lincei 74 (1983), 366-372.

6. __ On weak i-homotopy equivalences of modules, Rend. Accad. Naz. Lincei 76 (1984).

7. P. Hilton, Homotopy theory and duality, Notes on Math. and its Appl., Gordon \& Breach, New York, London and Paris, 1965.

8. I. Kaplansky, Infinite abelian groups, Univ. of Michigan Press, Ann Arbor, 1969.

9. E. Matlis, Injective modules over Noetherian rings, Pacific J. Math. 8 (1958), 511-528.

10. Z. Papp, On algebraically closed rings, Publ. Math. Debrecen 6 (1959), 311-327.

Faculty of Mathematics, University of IAsi, R - 6600, IAsi, Romania 